\title{
A Robust Reconfigurable Control Scheme Against Pose Estimation Induced Time Delays
}

\author{
Christoforos Kanellakis and George Nikolakopoulos
}

\begin{abstract}
Time delays are one of the most common problems when utilizing a visual sensor for pose estimation or navigation in aerial robotics. Such time delays can grow exponentially as a function of the scene's complexity and the size of the mapping during classical Simultaneous Localization and Mapping (SLAM) strategies. In this article, a robust reconfigurable control scheme against pose estimation induced time delays is presented. Initially, an experimental verification of the induced time delays via pose estimation is performed for the attitude problem of a hexacopter, while a switching time delay dependent modeling approach is formulated. In addition, a stability analysis algorithm is introduced in order to evaluate the maximum allowable time delays that the target system can handle for a given $L Q R$ controller. The varying nature of the time delays results in a switching system with the latency time to play the role of a switching rule, while simulation results are presented to outline the effects of the time-induced delays in hexarotor-based systems and finally evaluate the overall efficiency of the proposed control scheme.
\end{abstract}

\section{INTRODUCTION}

Recently, the area of Unmanned Aerial Vehicles (UAVs) has become a major field of research [1], [2]. In general, UAVs are distinguished for their ability to fly in low speeds, to stabilize their position, to hover over a target and to perform manoeuvres in close distances [3]. Nowadays, more and more UAVs are recruited for civilian applications in terms of surveillance and infrastructure inspection, thanks to their design that makes them quite powerful and agile. The main interest lies in introducing autonomous UAVs, by enabling 3D flights in unknown environments to explore autonomously and interact with the surrounding environment.

A fundamental ability that a self-exploring aircraft should possess is the perception and analysis of its surroundings [4]. To this end, computer vision techniques for Simultaneous Localization And Mapping (SLAM) [5] of the vehicle were employed [6].From a methodological perspective, along with the SLAM there exist Visual Odometry approaches that utilized a vision system for relative pose estimation. Many SLAM and Visual odometry [7] algorithms or combination of both, have been proposed with remarkable results, nevertheless with crucial processing and time constraints in their operation [8]. Moreover, UAVs inherently possess fast dynamics, leading to attitude/position control schemes with high update rates. As a consequence, to incorporate computer

The authors are with the Control Engineering Group, Department of Computer Science, Electrical and Space Engineering, Luleå University of Technology, SE-97187, Luleå, Sweden

Corresponding Author's email: chrkaneltu

This work has received funding from the European Unions Horizon 2020 Research and Innovation Programme under the Grant Agreement No.644128, AEROWORKS.

(C2016 IEEE vision algorithms into the control scheme of these vehicles, it is needed to ensure bounded time delays for feasible navigation. This need becomes more evident especially in flying UAVs, since in this case the pose estimation and the visual odometry induced time delays can deteriorate the performance of the overall system, even drive it to instability (crashes), a case that is different to the ground vehicles, where the existence of such time delays can only significantly reduce the cruising speed.

Regarding the problem of stability issues due to latencies introduced from computationally intensive computer vision algorithms, in [9] various techniques to deal with fusion of time delayed measurements were presented, based on Kalman filtering. In [10] an Extended Kalman filter (EKF) has been developed, where the state predictor was employed to estimate the delay between the camera and the inertial measurements. Similarly, in [11], the video stream was timestamped in order to determine the magnitude of the delays and afterwards, fused the data with inertial measurements with a modified EKF. Moreover, in [12] a filtered based position and velocity estimation, fusing inertial and vision measurements has been presented, while as discussed previously this approach was based also on an EKF. Finally, in [8] a stability analysis for a vision navigated autonomous UAV was described, where a hierarchical controller was designed considering a time scale decoupling between fast and slow dynamics. In contrast to the previous presented approaches, this article will focus on the problem of the attitude regulation based only on a stereoscopic camera system, while it will propose a novel approach in taking under consideration the induced time delays during the control design stage, based on an existing stereoscopic vision algorithm. In more details, the contribution of this article is triple. Firstly, a formal stability analysis scheme, being able to evaluate the maximum tolerated vision based time delay, that the UAV can handle will be established based on Linear Matrix Inequalities (LMIs). Secondly, a switching modeling framework will be derived, being able to take under consideration the time varying character of the vision based induced time delays. Thirdly, one of the specific aims of this article is to stress the fact that an on-line SLAM algorithm is not always applicable out of the box on UAVs, due to the numerous time delay stability issues. More specifically, as it will be presented and discussed the processing time of the pose estimation and odometry algorithms may exceed the response time of the aerial vehicle and thus directly deteriorate the performance of the vehicle, even driving it to instability. 
The rest of this article is structured as follows. In Section II the evidence of the pose estimation induced time delays will be presented through experimental flying results, while in Section IV the novel stability analysis framework towards the maximum anticipated delay that the UAV is able to tolerate will be presented, followed by a detailed switching modeling approach for taking under consideration the time varying nature of the time delays. In Section $\mathrm{V}$ simulation results that prove the efficiency of the proposed scheme are presented, while the conclusions and the overall discussion are being provided in Section VI.

\section{Visual SLAM INDUCED TIME DELAYS}

In this article, the Real Time Appearance based Mapping (RTABMap) [13] is utilized as the base for the SLAM algorithm using solely a stereo camera. This graph-based RGBD SLAM method consists of three modules: a) the frontend, b) the backend, and c) the final map representation. In short, during the frontend phase of the algorithm, the geometric information between the sensor and the environment are derived, while the backend part is dealing with the sensor's noise and other inherent uncertainties by employing optimization techniques. The full aerial setup considered, is a hexarotor UAV with the on board mounted stereo camera based visual sensor as depicted in Figure 1. More specifically,

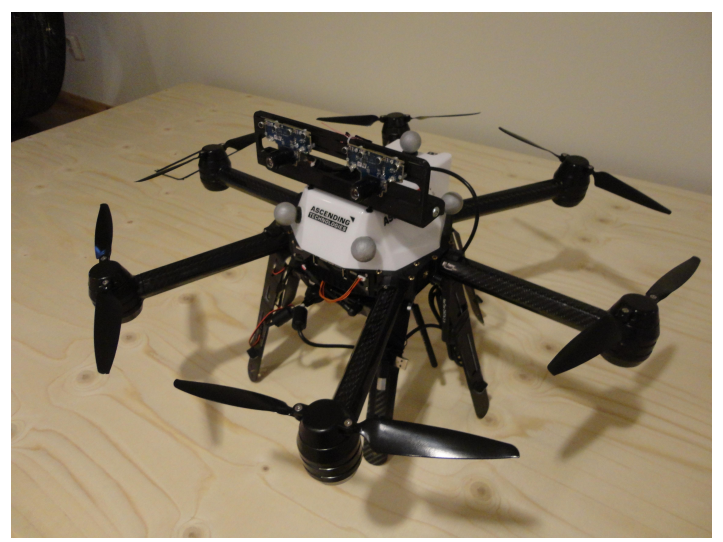

Fig. 1: AscTec Neo with the stereo camera onboard

the visual sensor employed for this study is a stereoscopic rig that consists of two PlayStation 3 EYE webcameras, with 30 frames per second at $640 \times 480$ pixels. Moreover, the cameras were disassembled from their casing and were fixed on an adhoc 3D printed base, in stereo canonical configuration with $11 \mathrm{~cm}$ baseline. It should be highlighted that the cameras were hardware synchronized by connecting their capture control hardware pins. Specific to this article, the pose estimation thread of the aforementioned RTABMap algorithm is being utilised, where the visual sensor is being considered as a black box sensor that will appropriately provide the pose estimation measurements to the utilized control scheme.

For controlling the attitude of the UAV, a generic attitude controller has been also incorporated, where for the initial stability analysis, the case of an LQR controller will be considered, as it will be presented in the sequel. In this case, the RTABMap is employed in this study to highlight the potential stability problems that come up in the integration of a robust and highly accurate mapping system on aerial platforms, while it should be mentioned that in this approach it has been assumed that the UAV is flying on no other navigation aids (e.g. IMU) apart from a stereo vision camera.

Initially, and for evaluating the performance of the RTABMap and specifically analysing the induced time delays, the UAV with the on-board stereo camera was manually operated in the LuleåUniversity of Technology FROST Flying Lab arena as presented in 2.

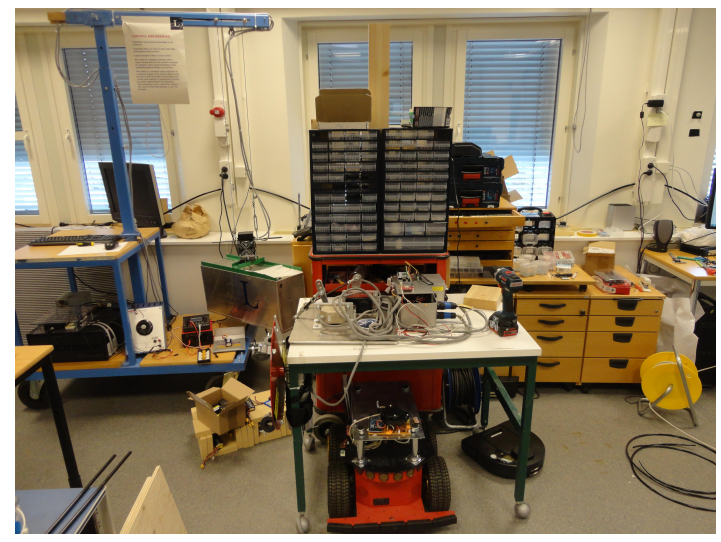

Fig. 2: Partial view of the flying environment utilized for the reconstruction

In this case, the SLAM algorithm RTABMap tracked the trajectory of the hexarotor by utilizing stereo visual odometry and provide 3D reconstruction of the environment (Figure 3) based on a computer with an Intel Core i7 CPU, 3.30GHz and $12 \mathrm{~Gb}$ RAM. The overall algorithmic implementation was made in the ROS (Robot Operating System [14]) framework.

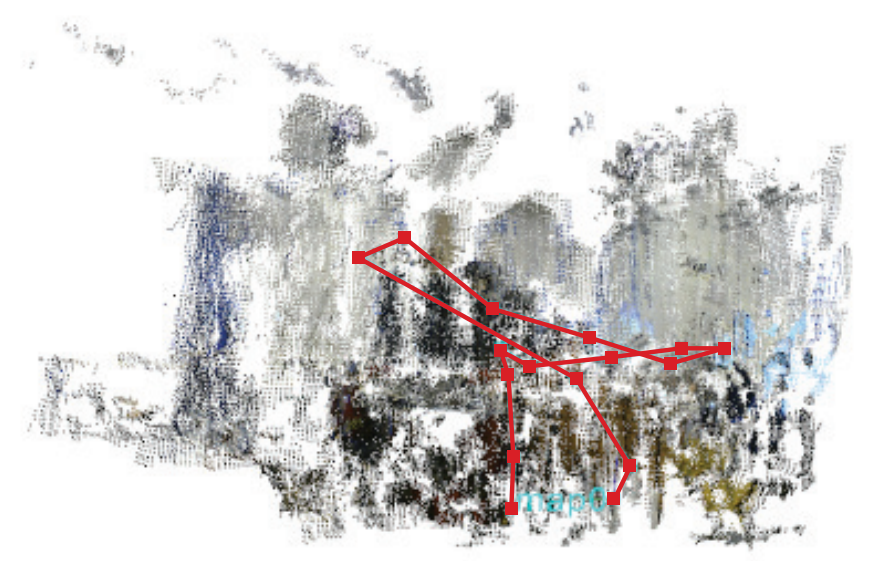

Fig. 3: Trajectory (red-highlighted lines) and 3D reconstruction in manual flight

Based on the previous experimental setup, it has been measured that the processing time for the pose estimation at 
every sample instant, induces time varying delays. It should be noted that the delay magnitude increases correspondingly to the population of the builded map. This varying time delay, is a factor that deteriorates the overall stability of the closed loop system and thus for improving the performance of the control scheme it should be taken under consideration. Even from a simple mapping case of the presented testing scene, these time delays can grow significantly and become even 10 times bigger. Thus, in Section III, a proper modeling of the UAV system to take under consideration the effect of the varying time delays and to evaluate the maximum time delay that the UAV can tolerate without turning unstable will be presented. Furthermore, this information will be also utilized in designing a switching control framework for achieving an overall performance improvement of the closed loop UAV's attitude problem.

\section{Hexarotor Modeling}

In this article the attitude regulation of the hexacopter UAV is examined when time delays are induced in the system. The nonlinear rotation dynamics of the aerial platform are decoupled from the translation ones and can be described by (1) [15]. This representation considers the torque induced aerodynamic resistance and the gyroscopic effect from the propellers. The states of the system are represented by the $[\phi, p, \theta, q, \psi, r]^{T}$ vector. To make the system platform independent, the inputs are chosen as the total aerodynamic force and torque, as well as the gyroscopic torque. Therefore, the system has 5 inputs $\left[U_{f}, U_{p}, U_{q}, U_{r}, \Omega_{r}\right]^{T}$, with $U_{f}$ to be the total thrust force, $U_{p}, U_{q}, U_{r}$ the roll, pitch, and yaw torques from the propeller aerodynamic effects respectively, while $\Omega_{r}$ is the disturbance torque produced by the gyroscopic torques from the propellers. The relation among these inputs and the velocities of the six motors $\Omega_{i}, i \in\{1, \cdots, 6\}$ is presented in equation 2 .

$$
\begin{aligned}
\dot{\phi} & =p+q \sin \phi \tan \theta+r \cos \phi \tan \theta \\
\dot{p} & =\frac{I_{y y}-I_{z z}}{I_{x x}} q r+\frac{J_{r}}{I_{x x}} q \Omega_{r}+\frac{l_{p}}{I_{x x}} U_{p} \\
\dot{\theta} & =q \cos \phi-r \sin \phi \\
\dot{q} & =\frac{I_{z z}-I_{x x}}{I_{y y}} p r-\frac{J_{r}}{I_{y y}} p \Omega_{r}+\frac{l_{p}}{I_{y y}} U_{q} \\
\dot{\psi} & =\frac{q \sin \phi}{\cos \phi}+\frac{r \cos \phi}{\cos \theta} \\
\dot{r} & =\frac{I_{x x}-I_{y y}}{I_{z z}} p q+\frac{U_{r}}{I_{z z}}
\end{aligned}
$$

where $I_{x x}, I_{y y}$ and $I_{z z}$ are the body inertia elements with respect to $X, Y$ and $Z$-axis, I $p$ and $J_{r}$ are the hexarotor's arm length and rotor's inertia respectively, while the adopted model parameters are shown in Table I.

$$
\begin{aligned}
& U_{f}=b\left(\Omega_{1}^{2}+\Omega_{2}^{2}+\Omega_{3}^{2}+\Omega_{4}^{2}+\Omega_{5}^{2}+\Omega_{6}^{2}\right) \\
& U_{p}=b l\left(-\frac{\Omega_{1}^{2}}{2}-\Omega_{2}^{2}-\frac{\Omega_{3}^{2}}{2}+\frac{\Omega_{4}^{2}}{2}+\Omega_{5}^{2}+\frac{\Omega_{6}^{2}}{2}\right) \\
& U_{q}=\frac{b l \sqrt[2]{3}}{2}\left(-\Omega_{1}^{2}+\Omega_{3}^{2}+\Omega_{4}^{2}-\Omega_{6}^{2}\right) \\
& U_{r}=d\left(-\Omega_{1}^{2}+\Omega_{2}^{2}-\Omega_{3}^{2}+\Omega_{4}^{2}-\Omega_{5}^{2}+\Omega_{6}^{2}\right) \\
& \Omega_{r}=-\Omega_{1}+\Omega_{2}-\Omega_{3}+\Omega_{4}-\Omega_{5}^{2}+\Omega_{6}^{2}
\end{aligned}
$$

TABLE I: AscTec Neo Parameters

\begin{tabular}{|c|c|}
\hline Par. & Value \\
\hline$m_{s}$ & $0.68 \mathrm{~kg}$ \\
\hline$I_{x x}$ & $0.0331 \mathrm{kgm}^{2}$ \\
\hline$I_{y y}$ & $0.0350 \mathrm{kgm}^{2}$ \\
\hline$I_{z z}$ & $0.0605 \mathrm{kgm}^{2}$ \\
\hline
\end{tabular}

\begin{tabular}{|c|c|}
\hline Par. & Value \\
\hline$I p$ & $0.2495 \mathrm{~m}$ \\
\hline$b$ & $6.8 \times 10^{-6} \mathrm{Ns}^{2}$ \\
\hline$d$ & $2.0673 \times 10^{-4} \mathrm{Nms}^{2}$ \\
\hline$J_{r}$ & $1.56 \times 10^{-3} \mathrm{kgm}^{2}$ \\
\hline
\end{tabular}

\section{Delay Dependent Switching Modeling And STABILITY PROOF}

\section{A. Delay Dependent Switching Modeling}

The linearized model of the hexarotor derived in section III is employed in the corresponding time delayed dynamics. Initially, the system is represented in the discrete state space using the following equations:

$$
\begin{aligned}
\mathbf{x}_{\mathrm{k}+1} & =\mathbf{A} \mathbf{x}_{\mathrm{k}}+\mathbf{B} \mathbf{u}_{\mathrm{k}} \\
\mathbf{y}_{\mathrm{k}} & =\mathbf{C} \mathbf{x}_{\mathrm{k}}
\end{aligned}
$$

where $\mathbf{x}_{\mathrm{k}} \in \Re^{6}, \mathbf{u}_{\mathrm{k}} \in \Re^{4}, \mathbf{y}_{\mathrm{k}} \in \Re^{6}$ with $\mathrm{k} \in \mathbb{Z}^{+}, \mathbf{C}=I_{6 \times 6}$ and the state space matrices $\mathbf{A}, \mathbf{B}$ defined as:

$$
\mathbf{A}=\left[\begin{array}{cccccc}
1 & T_{s} & 0 & 0 & 0 & 0 \\
0 & 1 & 0 & 0 & 0 & 0 \\
0 & 0 & 1 & T_{s} & 0 & 0 \\
0 & 0 & 0 & 1 & 0 & 0 \\
0 & 0 & 0 & 0 & 1 & T_{s} \\
0 & 0 & 0 & 0 & 0 & 1
\end{array}\right] \mathbf{B}=\left[\begin{array}{ccccc}
0 & 0 & 0 & 0 & 0 \\
0 & b_{1} & 0 & 0 & 0 \\
0 & 0 & 0 & 0 & 0 \\
0 & 0 & b_{2} & 0 & 0 \\
0 & 0 & 0 & 0 & 0 \\
0 & 0 & 0 & b_{3} & 0
\end{array}\right]
$$

where $b_{1}=\frac{I_{p}}{I_{x x} T_{s}}, b_{2}=\frac{I_{p}}{I_{y y} T_{s}}$ and $b_{3}=\frac{I_{p}}{I_{z z} T_{s}}$ and $T_{s}$ the sampling time.

Additionally to these system equations, an attitude controller needs to be integrated as it has been depicted in Figure 1 [16] and thus a state feedback Linear Quadratic Regulator (LQR) [17] can be designed as shown in (4), considering zero latency feedforward and feedback connections in the closed loop system by minimizing a quadratic cost function as described in (5):

$$
\begin{gathered}
\mathbf{u}_{\mathrm{k}}=-\mathbf{K} \mathbf{x}_{\mathrm{k}} \\
J_{\mathbf{u}}=\sum_{\mathrm{k}=1}^{\infty}\left[\mathbf{x}^{\mathrm{T}}(\mathrm{k}) \mathbf{Q} \mathbf{x}(\mathrm{k})+\mathbf{u}^{\mathrm{T}}(\mathrm{k}) \mathbf{R u}(\mathrm{k})\right]
\end{gathered}
$$

where $\mathbf{Q}$ and $\mathbf{R}$ are the quadratic weights of the cost function, weighting respectively the state deviation and the input.

The calculation of the LQR's gain $\mathbf{K}$ is a sufficient condition to guarantee the overall stability of the closed-loop system, while the corresponding discrete time state space model is also asymptotically stable, regardless of the stability 
of the open loop system keeping the modulus of the poles or eigenvalues of $\mathbf{A}$ smaller than one, while this analysis is true for a zero latency case. In the case of time delays, the closed loop poles of the system are varying towards the limits of the unitary circle and thus the bigger the time delay is, the easier the system approaches the instability region, provided a fixed control structure.

In the case that varying time delays are induced to the system from the pose estimation, multiple bounded delayed regions can be defined, as it can be observed from Figure 6, meaning that the maximum delay can be assumed known a priori (worst case scenario), while the notation of the stereo camera to controller time delay can be defined as: $\tau(k) \in \Re^{+}$. In a zero latency connection, meaning that $\tau=0$, the attitude controller, corresponds to a static output feedback defined as $\tilde{\mathbf{u}}(\mathrm{k})=\mathbf{K e}(\mathrm{k})=\mathbf{K}(\mathbf{r}(\mathrm{k})-\mathbf{y}(\mathrm{k}))$. However, since the amplitude of processing time on the visual sensor exceeds the system update rate response time, time delays cannot be neglected in the design of the controller. To this end, the applied control signal is $\mathbf{e}(\mathrm{k})=\mathbf{r}(\mathrm{k})-\mathbf{y}(\mathrm{k}-\tau(k))$ and for the case of the attitude regulation problem $\mathbf{r}$ is set to zero, while for simplicity in the notations we define $d(k)=\left\lceil\frac{\tau(k)}{T_{s}}\right\rceil$, with $d \in \mathbb{Z}^{+}$be the overall delay at sampled instance $k$ and $T_{s}$ the selected sampling period. If the overall time delay $d$ is time varying the resulting control law is provided by:

$$
\mathbf{u}_{\mathrm{k}}=\mathbf{K C y}(\mathrm{k}-d(\mathrm{k}))
$$

For the scope of the analysis, the state vector $\mathbf{x}(\mathrm{k})$ is transformed to an augmented version in order to include all the delays, as shown below [18]:

$$
\tilde{\mathbf{x}}_{\mathrm{k}+1}=\left[\mathbf{x}_{\mathrm{k}}^{T}, \mathbf{x}_{\mathrm{k}-1}^{T}, \ldots, \mathbf{x}_{\mathrm{k}-D}^{T}\right]^{T}
$$

with $\tilde{\mathbf{x}} \in \Re^{(D+1) \times(D+1)}$ and $D$ the maximum bound of the time delay. The augmented state space representation of the system at sample time $k$ can be described as in Equation 8:

$$
\begin{aligned}
\tilde{\mathbf{x}}_{\mathrm{k}+1} & =\tilde{\mathbf{A}} \tilde{\mathbf{x}}_{\mathrm{k}}+\tilde{\mathbf{B}} \mathbf{u}_{\mathrm{k}} \\
\mathbf{y}_{\mathrm{k}} & =\tilde{\mathbf{C}}_{\mathrm{a}} \tilde{\mathbf{x}}_{\mathrm{k}},
\end{aligned}
$$

where the matrices $\tilde{\mathbf{A}}, \tilde{\mathbf{B}}$ and $\tilde{\mathbf{C}}_{\mathrm{a}}$ are defined as [19]:

$$
\begin{gathered}
\tilde{\mathbf{A}}=\left[\begin{array}{ccccc}
\mathrm{A} & 0 & \ldots & 0 & 0 \\
\mathrm{I} & 0 & \ldots & 0 & 0 \\
0 & \mathrm{I} & \ldots & 0 & 0 \\
\vdots & \vdots & \vdots & \vdots & \vdots \\
0 & 0 & \ldots & \mathrm{I} & 0
\end{array}\right], \tilde{\mathrm{B}}=\left[\begin{array}{c}
\mathrm{B} \\
0 \\
0 \\
\vdots \\
0
\end{array}\right], \\
\tilde{\mathbf{C}}_{\mathrm{a}}=\left[\begin{array}{lllllll}
0 & \ldots & 0 & \mathrm{C} & 0 & \ldots & 0
\end{array}\right]
\end{gathered}
$$

where the matrix term $\mathbf{C}$, stems from the initial system and lies in $(a+1)^{\text {th }}$ column of $\tilde{\mathbf{C}}_{\mathrm{a}}(\mathrm{k})$, $\tilde{\mathbf{A}} \in \Re^{(D+1) \cdot n \times(D+1) \cdot n}, \tilde{\mathbf{B}} \in \Re^{(D+1) \cdot n \times m}$ and $\tilde{\mathbf{C}}_{\mathrm{a}}(\mathrm{k}) \in \Re^{(D+1) \cdot n \times n}$.
By combining (6) and (9), the state feedback closed loop discrete system can be summarized in Equations 10 and 12:

$$
\begin{aligned}
\tilde{\mathbf{x}}_{\mathrm{k}+1} & =\left(\tilde{\mathbf{A}}+\tilde{\mathbf{B}} \mathbf{K} \tilde{\mathbf{C}}_{\mathrm{a}}\right) \tilde{\mathbf{x}}_{\mathrm{k}} \\
\mathbf{y}_{\mathrm{k}} & =\tilde{\mathbf{C}}_{\mathrm{a}} \tilde{\mathbf{x}}_{\mathrm{k}},
\end{aligned}
$$

or

$$
\tilde{\mathbf{x}}_{\mathrm{k}+1}=\mathbf{A}_{i} \tilde{\mathbf{x}}_{\mathrm{k}+1}
$$

where (12) stems from the closed loop matrix $\tilde{\mathbf{A}}+\tilde{\mathbf{B}} \mathbf{K} \tilde{\mathbf{C}}_{\mathrm{a}}$, which can switch between any of its D+1 vertices (any of D different delays or no delay). Thereafter, the switched matrix becomes $\mathbf{A}_{\mathrm{i}}=\tilde{\mathbf{A}}+\tilde{\mathbf{B}} \mathbf{K}_{\mathrm{i}} \tilde{\mathbf{C}}_{\mathrm{i}}$, with $i: \mathbb{Z}^{+} \rightarrow I=0,1, \ldots, D$.

Under the assumption that at every time instance $k$ the latency time $d$ can be measured, and therefore the index of the switched-state is known, the system can be described as:

$$
x(k+1)=\sum_{i=0}^{D} \xi_{i}(k) A_{i} x(k),
$$

where $\xi(k)=\left[\xi_{0}(k), \ldots, \xi_{D}(k)\right]^{T}$ and $\xi=\left\{\begin{array}{l}1, \operatorname{mode}=A_{i} \\ 0, \operatorname{mode} \neq A_{i}\end{array}\right.$.

\section{B. Proof of Stability}

The stability of the switched system [20], in (13) is ensured if $D+1$ positive definite matrices $P_{i}, i=0, \ldots, D$ can be found that satisfy the following LMI:

$$
\begin{aligned}
{\left[\begin{array}{cc}
P_{i} & A_{i}^{T} P_{j} \\
P_{j} A_{i} & P_{j}
\end{array}\right] } & >0, \forall(i, j) \in I \times I \\
P_{i} & >0, \forall i \in I=\{0,1, \ldots, D\}
\end{aligned}
$$

Based on these $P_{i}$-matrices, it is feasible to calculate a positive Lyapunov function of the form $V(k, x(k))=x(k)^{T}\left(\sum_{i=0}^{D} \xi_{i}(k) P_{i}\right) x(k)$ whose difference $\Delta V(k, x(k))=V(k+1, x(k+1))-V(k, x(k)))$ is a negative function for all the $x(k)$-solutions of the switched system, thus ensuring the asymptotic stability of the system.

\section{Simulation Results}

\section{A. Stability analysis}

The corresponding simulation dynamics consider the system linearized around $\mathbf{x}_{0}=[0,0,0,0,0,0]^{T}$, assuming a sampling time of $T_{s}=0.001 \mathrm{~s}$. More specifically, the discrete matrices $\mathbf{A}, \mathbf{B}$ are depicted as it follows:

$$
\mathbf{A}=\left[\begin{array}{cccccc}
1 & 0.001 & 0 & 0 & 0 & 0 \\
0 & 1 & 0 & 0 & 0 & 0 \\
0 & 0 & 1 & 0.001 & 0 & 0 \\
0 & 0 & 0 & 1 & 0 & 0 \\
0 & 0 & 0 & 0 & 1 & 0.001 \\
0 & 0 & 0 & 0 & 0 & 1
\end{array}\right], \mathbf{B}=\left[\begin{array}{ccccc}
0 & 0 & 0 & 0 & 0 \\
0 & 0.0075 & 0 & 0 & 0 \\
0 & 0 & 0 & 0 & 0 \\
0 & 0 & 0.0071 & 0 & 0 \\
0 & 0 & 0 & 0 & 0 \\
0 & 0 & 0 & 0.041 & 0
\end{array}\right]
$$

Based on the stability proof provided in the previous Section, in Figure 4 it is presented the maximum allowable time delay that keeps the system stable, in the case that a fixed LQR controller is considered.

This Figure presents also the maximum eigenvalue of $\tilde{\mathbf{A}}$ as a function of the time delay for seven different values of the LQR. Indicatively, for the selected gains $K=$ $0.1 K_{L Q R}, K=0.2 K_{L Q R}, K=0.35 K_{L Q R}$, , the system 


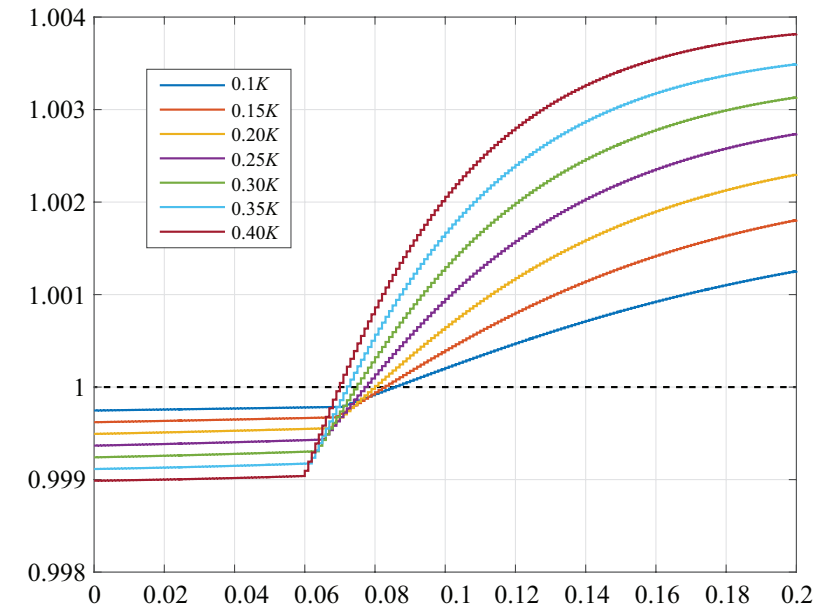

Fig. 4: Maximum allowable time delay versus various fixed LQR controllers

becomes unstable for delays greater than 0.085, 0.08 and 0.072 respectively, since $\left|\lambda_{\max } \tilde{\mathbf{A}} \geq 1\right|$.

It should be also highlighted that by decreasing the LQR gain $\mathbf{K}$ the system remains stable for greater delays but the performance is decreased as well, while the exact gains of $\mathbf{K}, \mathbf{Q}$ and $\mathbf{R}$ were:

$\mathbf{K}=\left[\begin{array}{cccccc}0 & 0 & 0 & 0 & 0 & 0 \\ 31.2794 & 2.8809 & 0 & 0 & 0 & 0 \\ 0 & 0 & 9.9405 & 1.67 & 0 & 0 \\ 0 & 0 & 0 & 0 & 3.1542 & 1.2368 \\ 0 & 0 & 0 & 0 & 0 & 0\end{array}\right]$

$\mathbf{R}=I_{5 x 5}$ presented in Section III, as it is indicated in the following Table II.

TABLE II: Time Delay vs Active LQR

\begin{tabular}{|c|c|}
\hline Delay (sec) & Switching Gain \\
\hline $0-0.04$ & $0.25 \times K_{L Q R}$ \\
\hline $0.04-0.06$ & $0.2 \times K_{L Q R}$ \\
\hline $0.06-0.09$ & $0.15 \times K_{L Q R}$ \\
\hline $0.09-0.2$ & $0.10 \times K_{L Q R}$ \\
\hline
\end{tabular}

In Figures 5 and 6 the responses for the rotation angles and the angular velocities are depicted correspondingly, while in the same Figures, the simulated results form the case of a fixed LQR gain of $\mathbf{K}$ are also depicted only for the first 25 sec, in order to retain the visibility of the obtained responses. Furthermore, in Figure 7 the corresponding switching signal for the selection of the active LQR controller is also presented.

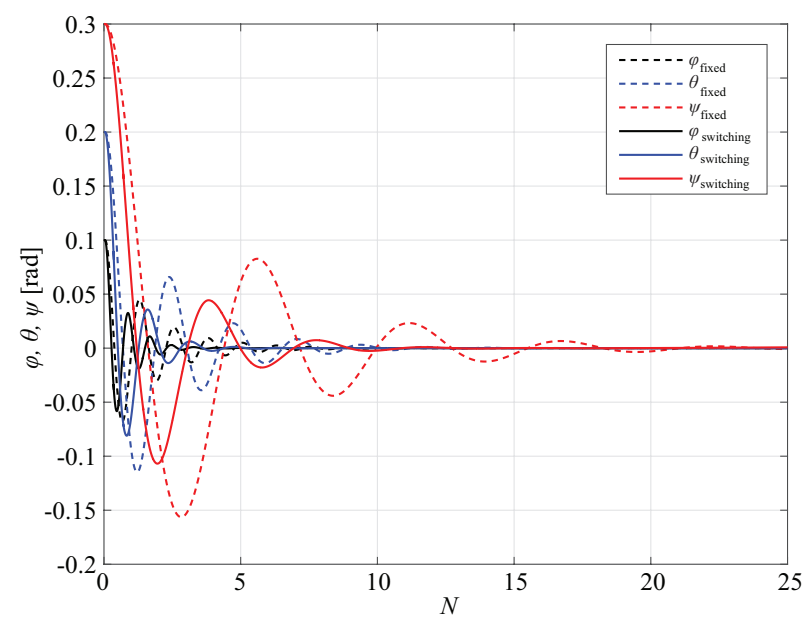

Fig. 5: Comparison of the Roll, Pitch and Yaw Responses for fixed and switching gain

From the obtained results it is obvious that the proposed switching scheme is able to provide a better regulation to the attitude problem with less overshoots and faster settling times, independently of the encountered time delays. Furthermore, based on the previous analysis, it should be highlighted that the system is able to tolerate now larger amount of time delays, when compared to the fixed controller case, while ensuring a good performance. In this approach, the pose estimation delays can be absorbed by the control scheme and thus singular to vision sensors systems can be used for the navigation of UAVs. At this point it should be also mentioned that the presented scheme can be directly extended to the translation estimation, with a corresponding position controller, since the induced time delays from the visual odometry have exactly the same characteristics in the pose and translation estimation.

\section{CONCLUSiOnS}

In this article a robust reconfigurable control scheme against vision based pose estimation induced time delays has stability limits that can be achieved from the stability analysis 


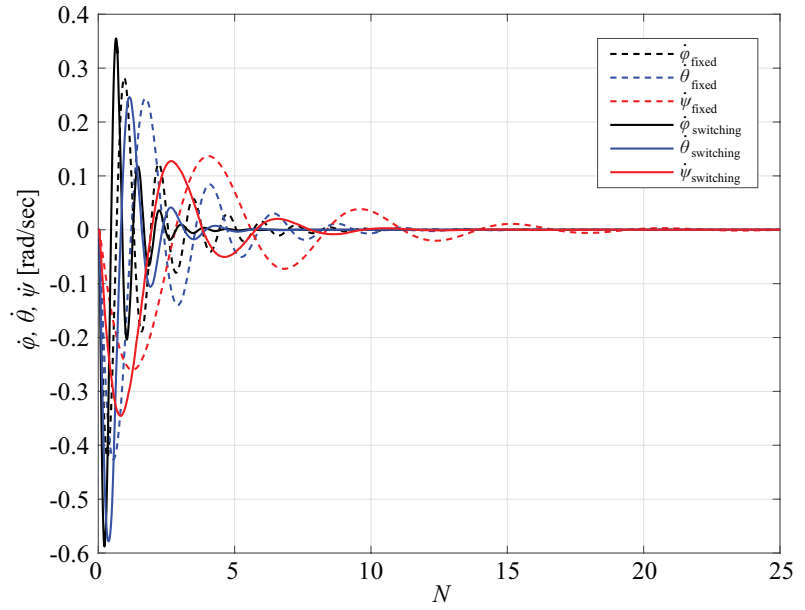

Fig. 6: Comparison of Angular Rate Responses for fixed and switching gain

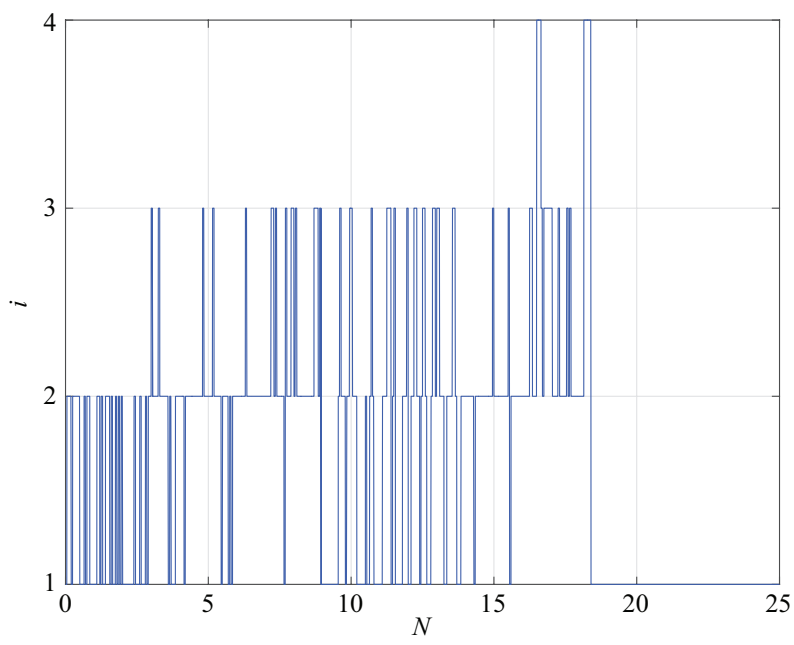

Fig. 7: LQR Switching Signal Selector response for fixed and switching gain

been considered. It has been presented, that varying latencies can drive the closed loop system to instability, without proper consideration in the control scheme. To overcome this bottleneck, a switching time delay dependent modeling approach has been proposed, while a stability analysis algorithm, based on the theory of LMIs has been established for evaluating the maximum time delay that the system is able to tolerate under the assumption of a fixed LQR controller. Based on this analysis, proper switching among fixed LQR controllers has been proposed as an approach in keeping the balance between performance and retaining stability, while simulation results have been presented that proved the efficiency of the proposed scheme.

\section{REFERENCES}

[1] K. P. Valavanis, Advances in unmanned aerial vehicles: state of the art and the road to autonomy. Springer Science \& Business Media, 2008, vol. 33 .
[2] K. Alexis, G. Nikolakopoulos, and A. Tzes, "Switching model predictive attitude control for a quadrotor helicopter subject to atmospheric disturbances," Control Engineering Practice, 2011, doi:10.1016/j.conengprac.2011.06.010.

[3] R. Austin, Unmanned aircraft systems: UAVS design, development and deployment. John Wiley \& Sons, 2011, vol. 54.

[4] B. Ludington, E. Johnson, and G. Vachtsevanos, "Augmenting uav autonomy," Robotics \& Automation Magazine, IEEE, vol. 13, no. 3, pp. 63-71, 2006.

[5] J. Fuentes-Pacheco, J. Ruiz-Ascencio, and J. M. Rendón-Mancha, "Visual simultaneous localization and mapping: a survey," Artificial Intelligence Review, vol. 43, no. 1, pp. 55-81, 2015.

[6] A. Bernatz and F. Thielecke, "Navigation of a low flying vtol aircraft with the help of a downwards pointing camera," in AIAA Guidance, Navigation and Control Conference, 2004.

[7] D. Nistér, O. Naroditsky, and J. Bergen, "Visual odometry," in IEEE Computer Society Conference on Computer Vision and Pattern Recognition (CVPR), vol. 1. IEEE, 2004, pp. I-652.

[8] A. Ramirez, E. S. Espinoza, L. G. Carrillo, S. Mondie, A. Garcia, and R. Lozano, "Stability analysis of a vision-based uav controller," Journal of Intelligent \& Robotic Systems, vol. 74, no. 1-2, pp. 69-84, 2014.

[9] T. D. Larsen, N. Andersen, O. Ravn, N. K. Poulsen et al., "Incorporation of time delayed measurements in a discrete-time kalman filter," in Proceedings of the 37th IEEE Conference on Decision and Control, vol. 4. IEEE, 1998, pp. 3972-3977.

[10] T. Zhang, W. Li, M. Achtelik, K. Kühnlenz, and M. Buss, "Multisensory motion estimation and control of a mini-quadrotor in an airground multi-robot system," in Robotics and Biomimetics (ROBIO), 2009 IEEE International Conference on. IEEE, 2009, pp. 45-50.

[11] M. Bošnak, D. Matko, and S. Blažič, "Quadrocopter hovering using position-estimation information from inertial sensors and a high-delay video system," Journal of Intelligent \& Robotic Systems, vol. 67, no. 1, pp. 43-60, 2012.

[12] F. Bourgeois, L. Kneip, S. Weiss, and R. Siegwart, "Delay and dropout tolerant state estimation for mavs," in Experimental Robotics. Springer, 2014, pp. 571-584.

[13] M. Labbe and F. Michaud, "Appearance-based loop closure detection for online large-scale and long-term operation," IEEE Transactions on Robotics, vol. 29, no. 3, pp. 734-745, 2013.

[14] "Robot operating system." [Online]. Available: http://www.ros.org/

[15] E. Capello, H. Park, B. Tavora, G. Guglieri, and M. Romano, "Modeling and experimental parameter identification of a multicopter via a compound pendulum test rig," in 2015 Workshop on Research, Education and Development of Unmanned Aerial Systems (RED-UAS). IEEE, 2015, pp. 308-317.

[16] S. Bouabdallah, A. Noth, and R. Siegwart, "Pid vs lq control techniques applied to an indoor micro quadrotor," in IEEE/RSJ International Conference on Intelligent Robots and Systems (IROS), vol. 3. IEEE, 2004, pp. 2451-2456.

[17] H. Kwakernaak and R. Sivan, Linear optimal control systems. Wileyinterscience New York, 1972, vol. 1.

[18] M. S. Mahmoud, Robust control and filtering for time-delay systems. CRC Press, 2000.

[19] A. Tzes, G. Nikolakopoulos, and I. Koutroulis, "Development and experimental verification of a mobile client-centric networked controlled system," European Journal of Control, vol. 11, no. 3, pp. 229-241, 2005.

[20] M. S. Mahmoud, Robust control and filtering for time-delay systems. CRC Press, 2000.

[21] S. Bouabdallah and R. Siegwart, "Full control of a quadrotor," in IEEE/RSJ international conference onIntelligent robots and systems (IROS). IEEE, 2007, pp. 153-158. 